Rev. Elev. Méd. vét. Pays trop., 1972, 25 (2): 317.324

\title{
Etude, en Côte d'Ivoire, de la croissance de taurillons N'Dama entretenus suivant divers modes d'embouche
}

\author{
J.-L. JOUVE $\left({ }^{*}\right)$, L. LETENNEUR $\left({ }^{*}\right)$
}

\begin{abstract}
RESUME
Etude pondérale comparative de divers lots de taurillons N'Dama entretenus pendant un an suivant des modalités différentes d'alimentation, dont certaines intensives. Les meilleurs résultats ont été cependant obtenus par les animaux laissés en permanence sur un pâturage naturel amélioré par le Stylosanthes et sans complémentation aucune.

Les auteurs concluent que c'est la durée du temps de pâture qui jone le rôle essentiel dans la croissance pondérale de ces animaux, ce qui est à la fois avantageux et économique.
\end{abstract}

Cette expérience, qui complète de précédents essais d'embouche a eu pour objectifs :

1. de comparer de façon systématique la croissance de taurillons N'Dama selon divers modes d'entretien (zéro pâturage - pâturage permanent - pâturage de jour avec ou sans complémentation).

2. d'étudier le prix de revient de la production de viande dans ces différentes conditions d'exploitation.

\section{MATERIEL ET METHODES}

L'expérience, qui débute le 5 juin 1969 , prend fin le 6 juin 1970 et dure donc un an.

\section{Animaux}

L'essai porte sur 40 taurillons de race N'Dama, âgés de 11 mois en moyenne au début de l'expérience, répartis par tirage au sort en 4 lots de 10 animaux.

(*) I.E.M.V.T., Centre de Recherches Zootechniques de Minankro, B.P. no 1152, Bouake, Côte d'Ivoire.
Tout au long de l'expérience, ils subissent les interventions suivantes: traitement contre les parasites gastro-intestinaux; douchages ixodicides hebdomadaires; vaccinations contre la peste bovine et la péripneumonie.

\section{Modalités d'entretien - Alimentation}

L'expérimentation comporte les modalités différentes d'entretien suivantes :

Lot I : Stabulation permanente;

Lot II : Pâturage de jour avec parcage de nuit et distribution d'aliment concentré;

Lot III : Pâturage de jour avec parcage de nuit sans distribution daliment concentré;

Lot IV : Pâturage permanent (jour et nuit) sans supplémentation.

Le lot I est entretenu en stabulation permanente. Les animaux reçoivent ad libitum une alimentation composée de Stylosanthes gracilis et de farines basses de riz.

Les lots II et III sont regroupés pendant la journée sur le même pâturage. Le soir, vers 
$16 \mathrm{~h} 30$, ils sont séparés et installés dans des parcs différents et seul le lot II reçoit alors des farines basses de riz comme complément alimentaire. Les animaux sont conduits matin, midi et soir à un abreuvoir.

Le lot IV, qui est entretenu en permanence sur un pâturage naturel amélioré en Stylosanthes, ne reçoit aucun aliment supplémentaire.

Tous les animaux ont à leur disposition des pierres à lécher comportant du phosphore, du chlorure de sodium et des oligo-éléments qui leur permettent d'équilibrer leurs besoins minéraux.

\section{Observations}

Elles sont relatives aux contrôles d'alimentation, à l'étude des gains de poids et à l'examen clinique.

\section{Consommations}

\section{Lot I}

La ration est distribuée matin et soir. Les quantités données et les refus sont pesés chaque jour. La consommation d'eau est mesurée au compteur.

Des échantillons de Stylosanthes gracilis et de farines de riz font l'objet d'analyses bromatologiques.

\section{Lot $I I$}

A chaque distribution, les farines de riz sont pesées. Il en est de même, tous les matins, pour les refus.

Le pâturage et l'abreuvement sont libres et ne font pas l'objet de mesures. Il en est de même pour les lots III et IV.

\section{Evolution pondérale}

Les animaux sont pesés individuellement le premier samedi de chaque mois à $8 \mathrm{~h}$ du matin. Ils sont à jeun au moment de la pesée : depuis la veille à $17 \mathrm{~h}$, tous les animaux sont gardés en parc où ils ne reçoivent ni alimentation ni abreuvement.

\section{Examen clinique}

Il fait l'objet d'un contrôle quotidien.

\section{RESULTATS}

a) Comportement pondéral

Les résultats généraux concernant les gains quotidiens moyens des quatre lots ont été les suivants :

Lot I : Stabulation libre $175 \mathrm{~g} / \mathrm{animal} / \mathrm{j}$.

Lot II : Pâturage de jour complémenté $330 \mathrm{~g} /$ animal $/ \mathrm{j}$.

Lot III : Pâturage de jour non complémenté $241 \mathrm{~g} /$ animal $/ \mathrm{j}$.

Lot IV : Pâturage permanent non complémenté $393 \mathrm{~g} /$ animal/j.

L'évolution des poids et celle des croîts quotidiens moyens sont exprimées dans les tableaux I et II et illustrées par le graphique I.

\section{b) Consommation}

\section{Lot $I$}

\section{a) farine basse de riz}

Compte tenu du fait que pendant les 106 premiers jours les animaux n'ont eu à leur disposition que du Stylosanthes gracilis, la consommation journalière par animal a été :

- du 8 octobre au 5 novembre de $0,5 \mathrm{~kg}$ soit $0,449 \mathrm{~g}$ de matière sèche et $62 \mathrm{~g}$ de matière azotée digestible et 0,46 UF;

- du 6 octobre 1969 au 7 mai 1970, de $1 \mathrm{~kg}$ de farine soit $0,898 \mathrm{~g}$ de matière sèche et $24 \mathrm{~g}$ de matière azotée digestible et 0,92 UF.

\section{b) Stylosanthes gracilis}

Pour les différentes périodes, les consommations journalières sont notées tableau III.

c) Indices de consommation

Pour les différentes périodes, les indices de consommation sont indiqués dans le tableau IV.

\section{Lot II}

Seule la consommation des farines de riz distribuées a pu être contrôlée. Elle est donnée dans le tableau $\mathrm{V}$.

\section{Lot III et IV}

Les animaux de ces lots sont entretenus au pâturage. Les consommations ne sont pas mesurées. 
TABLEAU $\mathrm{N}^{\circ}$ I

Evolution des poids moyens

\begin{tabular}{|c|c|c|c|c|c|c|c|c|c|c|c|c|c|}
\hline Dates & 8.6 & 5.7 & 2.8 & 6.9 & 4.10 & 1.11 & 6.12 & 3.1 & 6.2 & 7.3 & 4.4 & 5.5 & 6.6 \\
\hline Lot I & $\begin{array}{r}118,7 \\
\pm 14,0\end{array}$ & $\begin{array}{r}123,3 \\
\pm \quad 14,0\end{array}$ & $\begin{array}{r}128,4 \\
\pm 15,0\end{array}$ & $\begin{array}{r}135,4 \\
+16,4\end{array}$ & $\begin{array}{r}137,7 \\
\pm 16,7\end{array}$ & $\begin{array}{r}133,2 \\
\pm 16,5\end{array}$ & $\begin{array}{r}144,4 \\
+\quad 17,8\end{array}$ & $\begin{array}{r}152,8 \\
\pm \quad 19,0\end{array}$ & $\begin{array}{r}165,9 \\
+\quad 18,3\end{array}$ & $\begin{array}{r}176,1 \\
\pm \quad 19,4\end{array}$ & $\begin{array}{r}175,8 \\
\pm 18,7\end{array}$ & $\begin{array}{r}173,2 \\
\pm \quad 18,6\end{array}$ & $\begin{array}{r}180,3 \\
\pm \quad 21,3\end{array}$ \\
\hline Lot II & $\begin{array}{r}137,6 \\
\pm \quad 19,3\end{array}$ & $\begin{array}{r}139,5 \\
\pm 20,0\end{array}$ & $\begin{array}{r}159,2 \\
\pm 21,7\end{array}$ & $\begin{array}{r}175,5 \\
\pm \quad 23,0\end{array}$ & $\begin{array}{r}186,8 \\
+\quad 23,8\end{array}$ & $\begin{array}{r}191,1 \\
\pm \quad 23,0\end{array}$ & $\begin{array}{r}194,7 \\
\pm \quad 21,2 \\
\end{array}$ & $\begin{array}{r}203,1 \\
\pm \quad 23,3 \\
\end{array}$ & $\begin{array}{r}207,7 \\
\pm \quad 21,5 \\
\end{array}$ & $\begin{array}{r}220,3 \\
+\quad 22,4 \\
\end{array}$ & $\begin{array}{r}230,5 \\
\pm \quad 22,6\end{array}$ & $\begin{array}{r}241,1 \\
\pm \quad 22,5\end{array}$ & $\begin{array}{r}253,6 \\
\pm \quad 21,0\end{array}$ \\
\hline Lot III & $\begin{array}{r}127,1 \\
\pm \quad 11,7\end{array}$ & $\begin{array}{r}131,6 \\
\pm \quad 13,1\end{array}$ & $\begin{array}{r}146,2 \\
\pm \quad 14,0 \\
\end{array}$ & $\begin{array}{r}158,0 \\
\pm \quad 15,1\end{array}$ & $\begin{array}{r}167,6 \\
+\quad 15,5\end{array}$ & $\begin{array}{r}168,2 \\
\pm \quad 14,1\end{array}$ & $\begin{array}{r}170,6 \\
+\quad 14,5\end{array}$ & $\begin{array}{r}180,2 \\
\pm \quad 15,8\end{array}$ & $\begin{array}{r}187,4 \\
\pm \quad 17,0 \\
\end{array}$ & $\begin{array}{r}196,0 \\
+\quad 17,9\end{array}$ & $\begin{array}{r}196,8 \\
+\quad 17,8\end{array}$ & $\begin{array}{r}201,7 \\
\pm 19,3\end{array}$ & $\begin{array}{r}211,8 \\
\pm 19,4\end{array}$ \\
\hline Lot IV & $\begin{array}{r}114,0 \\
+\quad 12.9\end{array}$ & $\begin{array}{r}117,2 \\
\pm 13,6\end{array}$ & $\begin{array}{r}134,4 \\
\pm \quad 14,8\end{array}$ & $\begin{array}{r}152,6 \\
\pm 18,3\end{array}$ & $\begin{array}{r}161,9 \\
+19,4\end{array}$ & $\begin{array}{r}167,0 \\
\pm \quad 20,6\end{array}$ & $\begin{array}{r}178,7 \\
+\quad 23,1\end{array}$ & $\begin{array}{r}190,2 \\
\pm \quad 24,7\end{array}$ & $\begin{array}{r}205,9 \\
\pm \quad 26,0\end{array}$ & $\begin{array}{r}212,7 \\
\pm \quad 26,3\end{array}$ & $\begin{array}{r}216,8 \\
\pm 25,8\end{array}$ & $\begin{array}{r}237,0 \\
+\quad 25,8\end{array}$ & $\begin{array}{r}251,8 \\
\pm \quad 25,8\end{array}$ \\
\hline
\end{tabular}

TABLEAU $N^{\circ}$ II

Evolution des gains de poids

\begin{tabular}{|c|c|c|c|c|c|c|c|c|c|c|c|c|c|}
\hline \multicolumn{2}{|c|}{ D a t e s } & \multicolumn{2}{|l|}{$18-6$} & \multicolumn{2}{|c|}{$6-9$} & \multicolumn{2}{|c|}{$1-11$} & \multicolumn{2}{|c|}{$3-1$} & $6-2$ & \multicolumn{2}{|c|}{$4-4$} & $5-5$ \\
\hline Lot & $\begin{array}{l}\text { Croît } \\
\text { journalier }\end{array}$ & 270,5 & 182,1 & 200 & 82 & $-160,7$ & 320 & 300 & 385 & 352 & -11 & -90 & 222 \\
\hline I & $\begin{array}{l}\text { Croît } / j \\
\text { cumulể }\end{array}$ & 270,5 & 215,5 & 208,7 & 175,9 & 106,6 & 150,2 & 171,3 & 202,5 & 219 & 197 & 171 & 175 \\
\hline Lot & $\begin{array}{l}\text { Croît } \\
\text { journalier }\end{array}$ & 111,7 & 703,6 & 465,7 & 403,6 & 153,6 & 102,8 & 300 & 135 & 434 & 364,2 & 365 & 390 \\
\hline II & $\begin{array}{l}\text { Crô̂t } / \mathrm{j} \\
\text { cumulế }\end{array}$ & 111,7 & 480 & 474 & 455,5 & 393,4 & 333,9 & 329,1 & 300,8 & 315,6 & 320,3 & 324 & 330 \\
\hline Lot & $\begin{array}{l}\text { Croît } \\
\text { journalier }\end{array}$ & 264,7 & 521,4 & 337,1 & 342,9 & 21,4 & 68,5 & 342,9 & 212 & 296,5 & 28,6 & 169 & 316 \\
\hline III & $\begin{array}{l}\text { Croît/j } \\
\text { cumulé }\end{array}$ & 264,7 & 424,4 & 386,2 & 375 & 302,1 & 254,3 & 266,8 & 258,7 & 262,9 & 240,3 & 234 & 241 \\
\hline Lot & $\begin{array}{l}\text { Croît } \\
\text { journalier }\end{array}$ & 188,2 & 614,3 & 520 & 332,1 & 182,1 & 334,2 & 410,7 & 462 & 234,4 & 146,4 & 695 & 462,5 \\
\hline IV & $\begin{array}{l}\text { Croît } / \mathbf{j} \\
\text { cunulế }\end{array}$ & 195,2 & 453,3 & 482,5 & 443,5 & 389,7 & 378,3 & 382,9 & 394,4 & 376,7 & 354,5 & 386 & 392,6 \\
\hline
\end{tabular}




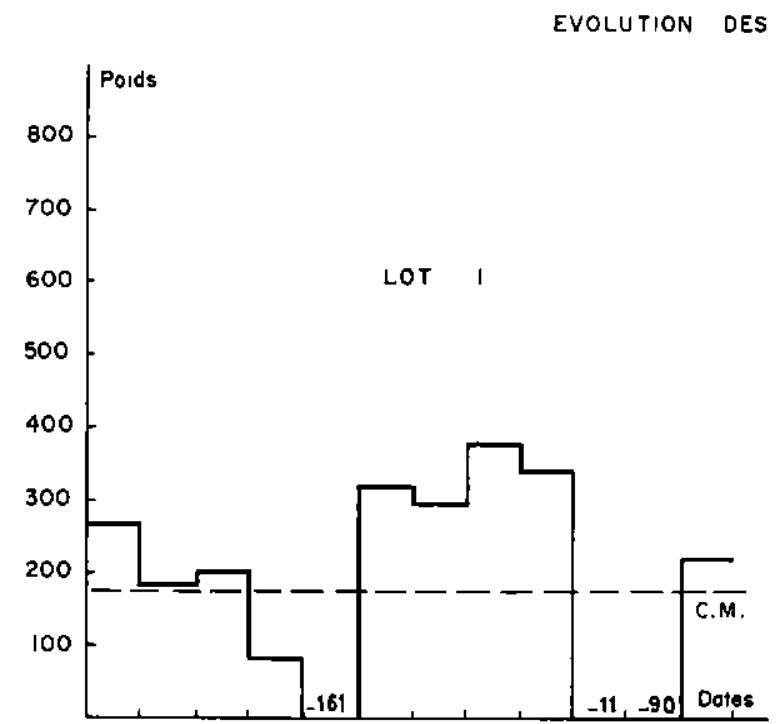

$\begin{array}{llllllllllll}5.7 & 2.8 & 69 & 4.10 & 1.11 & 6.12 & 31 & 6.2 & 7.3 & 4.4 & 55 & 6.6\end{array}$

GAINS DE POIOS
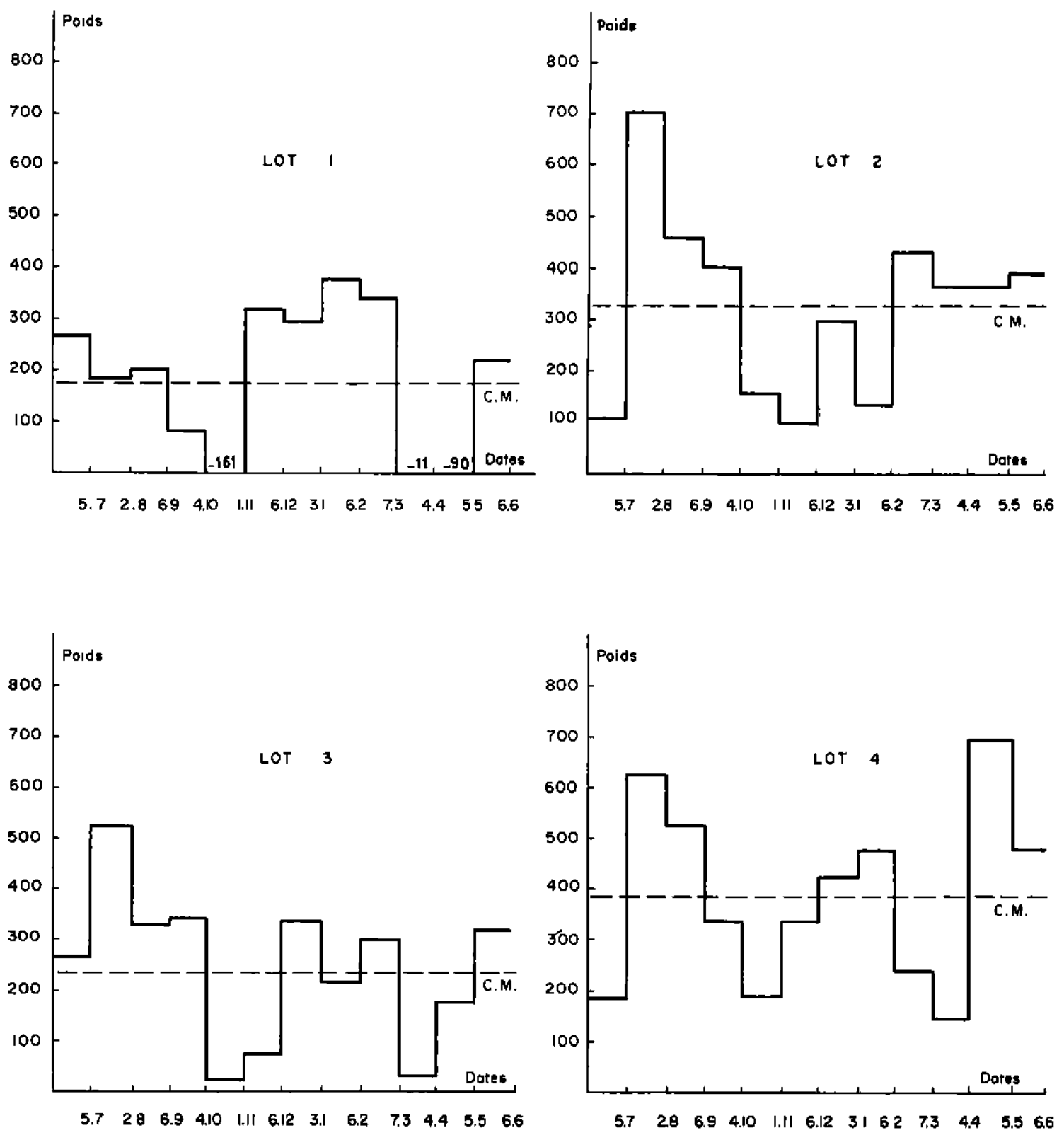
TABLEAU $\mathrm{N}^{\circ}$ III

Consomations - Stylosanthes gracilis

\begin{tabular}{|r|c|c|c|c|}
\hline Périodes & Quantités/anımal & M.s./animal & U.F./anımal & M.A.D./anima1 \\
\hline $23.6-29.6$ & 11,5 & 2,16 & 1,4 & 220 \\
$30.6-27.7$ & 13,3 & 3,21 & 2,1 & 315 \\
$28.7-31.8$ & 15,0 & 3,91 & 2,5 & 347 \\
$1.9-28.9$ & 13,8 & 3,36 & 2,12 & 250 \\
$29.9-2.1$ & 14,1 & 3,24 & 1,92 & 286 \\
$3.11-30.11$ & 16,2 & 3,11 & 1,87 & 220 \\
$1.12-28.12$ & 14,5 & 3,40 & 2,20 & 230 \\
$29.12-1.2$ & 13,9 & 4,6 & 3,03 & 334 \\
$2.2-2.3$ & 13,4 & 6,5 & 3,3 & 357 \\
$3.3-5.4$ & 7,3 & 4,09 & 2,3 & 212 \\
$6.4-26.4$ & 8,8 & 4,97 & 2,8 & 256 \\
$27.4-7.6$ & 13,4 & 4,5 & 4,2 & 389 \\
\hline
\end{tabular}

TABLIAU N ${ }^{\circ}$ IV

Indices de consommation

\begin{tabular}{|c|c|c|c|}
\hline Péruodes & U.E./animal/j & Gain de poids & I.C. \\
\hline $18.6-5.7$ & 1,4 & 270,5 & 5,1 \\
$6.7-2.8$ & 2,1 & 182,1 & 11,5 \\
$3.8-6.9$ & 2,5 & 200 & 12,5 \\
$7.9-4.10$ & 2,12 & 82 & 25,8 \\
$5.10-1.11$ & 2,38 & $-160,7$ & - \\
$2.11-6.12$ & 2,79 & 320 & 8,7 \\
$7.12-3.1$ & 3,12 & 300 & 10,4 \\
$4.1-6.2$ & 3,95 & 385 & 10,2 \\
$7.2-7.3$ & 4,62 & 352 & 13,1 \\
$8.3-4.4$ & 3,22 & -11 & - \\
$5.4-5.5$ & 6,94 & -90 & - \\
$6.5-6.6$ & 5,12 & 222 & 23,0 \\
\hline
\end{tabular}

TABLEAU $\mathrm{N}^{\circ} \mathrm{V}$

Consonmations - Farines de riz

\begin{tabular}{|c|c|c|c|c|}
\hline Périodes & $\begin{array}{c}\text { Quantı té/ } \\
\text { animal/j }\end{array}$ & M.S. & U.F. & M.A.D. \\
\hline $23.6-29.6$ & 0,47 & 0,42 & 0,43 & 58,2 \\
$30.7-27.7$ & 0,84 & 0,75 & 0,77 & 104,1 \\
$28.7-31.8$ & 0,97 & 0,87 & 0,89 & 120,2 \\
$1.9-28.9$ & 1,0 & 0,89 & 0,92 & 124,0 \\
$29.9-2.11$ & 0,95 & 0,85 & 0,87 & 117,8 \\
$3.11-30.11$ & 0,94 & 0,84 & 0,86 & 116,5 \\
$1.12-28.12$ & 0,98 & 0,88 & 0,90 & 121,5 \\
$29.12-1.2$ & 1,13 & 1,01 & 1,03 & 140,1 \\
$2.2-2.3$ & 1,10 & 0,98 & 1,01 & 136,4 \\
$3.3-5.4$ & 1,06 & 0,95 & 0,97 & 131,4 \\
$6.4-26.4$ & 1,10 & 0,98 & 1,01 & 136,4 \\
$27.4-7.6$ & 1,10 & 0,98 & 1,01 & 136,4 \\
\hline
\end{tabular}




\section{DISCUSSIONS}

L'examen des résultats permet les remarques suivantes :

1. Les animaux entretenus en parc d'embouche ont une croissance médiocre et des indices de consommation élevés.

Pendant la mauvaise saison, ils souffrent gravement de la faible valeur bromatologique de l'herbe distribuée; ce sont les seuls à perdre du poids;

2. Les animaux au pâturage se ressentent moins des variations de valeur alimentaire de l'herbe, à condition toutefois de disposer d'un temps de pâture suffisamment long: le lot IV au pâturage permanent conserve une croissance convenable, pendant les mois critiques (octobre à décembre et février-mars) alors que le lot III au pâturage de jour a une croissance presque nulle pendant les mêmes périodes;
3. La supplémentation au pâturage a un effet favorable sur la croissance pendant les périodes de disette. Son influence est moins nette lorsque le pâturage est de bonne qualité (comparaison des lots II et III d'une part et II et IV d'autre part).

Le modèle technique le meilleur semblerait donc être le pâturage permanent complémenté pendant la saison sèche.

\section{CALCUL ECONOMIQUE}

Nous prendrons comme valeur de référence, pour le calcul, les chiffres déjà mentionnés par les précédentes expériences.

Le bilan chiffré de l'essai est établi dans le tableau VI.

TABLEAU $N^{\circ} V I$

Bilan économique F C.F.A.

\begin{tabular}{|c|c|c|c|c|}
\hline & Lot I & Lot II & Lot III & Lot IV \\
\hline Traitement & $\begin{array}{l}\text { Stabulation } \\
\text { permanente }\end{array}$ & $\begin{array}{l}\text { Pâturage } \\
\text { diurne } \\
\text { complëmenté }\end{array}$ & $\begin{array}{l}\text { Pâturage } \\
\text { diurne non } \\
\text { complémenté }\end{array}$ & $\begin{array}{l}\text { Pâturage } \\
\text { permanent non } \\
\text { complëmentê }\end{array}$ \\
\hline Poids Initıal (kg) & 1187 & 1376 & 1271 & 1140 \\
\hline Poids final $(\mathrm{kg})$ & 1803 & 2536 & 2118 & 2. 518 \\
\hline Variation absolue (kg) & 616 & 1160 & 847 & 1378 \\
\hline Gain moyen / $\mathrm{j}(\mathrm{g})$ & 175 & 330 & 241 & 392 \\
\hline Coût de 1'alimentation & $45 \quad 092$ & 32964 & 11874 & 11874 \\
\hline Interventions - soins & 6000 & 6000 & 6000 & 6000 \\
\hline Main-d'oeuvre & 12775 & 31935 & 31935 & 12775 \\
\hline Amortissements & 26600 & 20000 & 20000 & 15000 \\
\hline Intërêt & 4600 & 4600 & 4600 & 4400 \\
\hline Total des charges & 95067 & 95499 & 74409 & 50049 \\
\hline Prix de vente / kg vif & 125 & 125 & 125 & 125 \\
\hline Valeur ajoutëe & 77000 & 145000 & 105875 & 172250 \\
\hline Marge nette & perte $=18.067$ & 49501 & 31466 & 122201 \\
\hline
\end{tabular}

\section{Conclusion}

Cette expérience avait pour but de déterminer les possibilités de croissance de taurillons de race N'Dama selon divers modes d'exploitation.
Elle permet de dégager les conclusions suivantes :

1. Le comportement des taurillons de race N'Dama et leur réponse à l'embouche varie avec les conditions d'entretien; 
2. L'entretien au pâturage permanent. sans supplémentation aucune, permet le maximum de croissance pondérale. Il réduit au minimum le temps nécessaire aux soins et aux manipulations des animaux;

3. L'entretien au pâturage de jour conduit à la limitation des niveaux de consommation. Cette restriction a un effet défavorable sur la croissance pondérale;

4. La supplémentation de la ration alimentaire prise au pâturage n'est justifiée qu'en période critique, fin de saison sèche par exemple;
5. L'alimentation en zéro pâturage à partir de prairie de fauche n'a pas permis de bons gains de poids dans les conditions de l'expérience;

6. La rentabilité de l'embouche est fonction du mode d'exploitation choisi. La technique la plus rentable, à la fois par la faiblesse des charges et l'importance de la valeur ajoutée par l'embouche est l'entretien au pâturage permanent sans supplémentation.

\section{RESUME}

Les auteurs ont étudié la croissance pondérale de divers lots de taurillons N'Dama, entretenus pendant une année suivant des modalités d'alimentation diverses. Ils ont constaté que les résultats les meilleurs ont été obtenus par les animaux laissés nuit et jour sur pâturage nature] amélioré au Stylosanthes mais sans complémentation aucune.

Les taurillons entretenus en stabulation permanente, dans les conditions classiques d'embouche intensive n'ont eu qu'un croît médiocre avec un indice de consommation élevé.

De même, les taurillons entretenus pendant la journée sur le même pâturage et enfermés la nuit, avec ou sans alimentation supplémentaire n'ont eu que des croîts nettement inférieurs aux premiers.

Il semble que, pour les animaux de cette race et dans les conditions où se sont déroulées ces observations, la longueur du temps de pâture dont peuvent disposer les animaux joue un rôle essentiel dans leur croissance pondérale, ce qui est à la fois avantageux et très économique.

\section{SUMMARY}

\section{Study of growth of N'Dama bull-calf differently fattened, in Ivory Coast}

The growth of various N'Dama young bulls lots differently fed during a year is studied.

The best results are obtained with cattle kept night and day on natural pasture, improved with Srylosanthes, but without any feed supplement.

Bull calves, in feedlot with intensive feeding had only a moderate growth with a low food conversion efficiency.

In the same way, the bulls kept during the day on the same pasture and confined during the night, with or without feed supplement, gained less than the first lot.

For this cattle breed and with the conditions of these observations, the duration of pasture plays an essential part in the: liveweight gain, which is at one and the same time advantageous and very economic.

\section{RESUMEN}

Estudio, en Costa de marfil, del crecimiento de novillos N'Dama según varios tipos de engorde

Los autores estudaron el crecimiento ponderal de varios lotes de novillos N'Dama, sometidos durante un año a varios tipos de alimentación. Se obtuvieron los mejores resultados con animales quedando durante la noche y el dia sobre pasto natural mejorado con Stylosanthes pero sin ningún aditivo.

Los novillos mantenidos en estabulación permanente, en las condiciones clásicas de engorde intensivo, no tuvieron más que un aumento mediano de peso con un indice de consumo elevado. 
Igualmente, los novillos mantenidos durante el dia sobre el mismo pasto y encerrados durante la noche, con o sin aditivos, no tuvieron más que incrementos terminantemente inferiores a los primeros.

En lo concerniente a los animales de esta raza y en las condiciones de realización de estas observaciones parece que la duración del pastoreo de los animales desempeña un papel esencial en su crecimiento ponderal, lo que es a la vez ventajoso y muy económico. 